\title{
Topical Therapies for Oral Lichen Planus Management and their Efficacy: A Narrative Review
}

\author{
Jose' Bagan $^{1, *}$, Domenico Compilato ${ }^{2}$, Carlo Paderni $^{2}$, Giuseppina Campisi ${ }^{2}$, Vera Panzarella ${ }^{2}$, Maria \\ Picciotti $^{3}$, Guido Lorenzini ${ }^{3}$ and Olga Di Fede ${ }^{2}$
}

${ }^{l}$ Service of Stomatology, University General Hospital of Valencia, Valencia, Spain; ${ }^{2}$ Sector of Oral Medicine “V. Margiotta”, Section of Odontostomatologic Sciences, Dept. Surgical and Oncological Disciplines, University of Palermo, Palermo, Italy; ${ }^{3}$ Tuscan School of Dental Medicine, University of Florence and Siena, Department of Oral Pathology University of Siena, Italy

\begin{abstract}
Oral Lichen Planus (OLP) is a chronic inflammatory condition implicating T cell-mediated cytotoxicity, and involving oral mucosal surfaces. Several therapeutic regimens have been evaluated to treat OLP and pain related, but often without high level of evidence. Topical formulations are the favourite for the majority of cases; bioadhesive formulations have been considered very useful and practical for local drug delivery in oral mucosa, due to the increased residence time on the oral mucosa of the dosage forms and better therapeutic efficacy.

In this narrative review, authors try to illustrate the current topical managements for OLP from the accessible literature on this topic. Steroids are very helpful in discomfort and making better quality of life: they are considered the first-line treatment even if they could cause secondary candidosis, and sometimes bad taste, nausea, dry mouth, sore throat or swollen mouth.

Other substances or devices by topical administration are adopted especially when the first line approach is refractory. This is the case when retinol with its synthetic and natural analogues (retinoids), hyaluronic acid, or Aloe Vera are chosen.

Recent topical applications for OLP therapy include phototherapy and low/high energy pulsing light; the treatment with extracorporeal photochemotherapy is also reasonable and promising.

Finally, calcineurin inhibitors (i.e. cyclosporine, tacrolimus and pimecrolimus), antioxidant and biologics (i.e alefacept, efalizumab, basiliximab, TNF- $\alpha$ inhibitors - infliximab, rituximab) may be alternative approaches when OLP does not respond to the standard protocols.

In this scenario, there are several studies on molecules different from glucocorticosteroids, but not sufficient or statistically adequate to justify their evidence-based use in OLP; large randomized placebo controlled trials are required to evaluate the safety and effectiveness of these non conventional therapies.

In conclusion, since OLP is a chronic disease and requires long-term management, the dental/medical practitioner, who treats OLP patients, needs to know the natural history of OLP, how to monitor, and how to treat, taking in account all of the available modalities conventional and not, with pros and cons.
\end{abstract}

Keywords: Oral Lichen Planus, topical therapy, corticosteroids, aloe vera, retinoids, hyaluronic acid, phototherapy, laser therapy.

\section{INTRODUCTION}

Lichen planus (LP) is an inflammatory mucocutaneous condition involving $\mathrm{T}$ cell-mediated cytotoxicity for reasons not yet definitively clarified. It affects skin and mucous membranes of stratified squamous epithelium with different clinical features [1, 2].

Sometimes, LP could be present in concomitant areas, with the epithelium being common to the other anatomic sites: oral, genital, nasal mucosa, and nails, rarely conjunctiva, larynx, and esophagus [3-5]. It has been also described a lichen planopilaris that involves the hair follicles resulting in scarring alopecia [6].

Oral mucosal lesions occur in 50 to $70 \%$ of the patients with LP. Oral lichen planus (OLP) affects $2 \%$ of the population with the highest incidence in women (2: 1), and the most affected age ranges from 40-70 years [7-22].

Current practice for the treatment of OLP (Table 1) seeks to ameliorate the symptoms and discomfort, to improve the normal functions (i.e. eating and speaking) and quality of life. In general, reticular and plaque forms do not necessitate a therapy, but only symptomatic forms need to be treated, and management is aimed to

*Address correspondence to this author at the Oral Medicine- Valencia University, Service of Stomatology. University General Hospital. Valencia. Avda de Tres Cruces s/n 46014 - Valencia, Spain;

Tel:/Fax: 34 961972127; E-mail: bagan@uv.es monitor the lesions and to reduce the frequency and seriousness of acute forms (Figs. 1, 2). Firstly, the elimination of irritating factors (e.g. maloccluded/fractured teeth, scarce oral hygiene, voluptuary habits) should be counselled.

Evaluation of effectiveness in OLP treatment strategy is complicated by many factors: patient's psychological profile and medical/pharmacological history (e.g. side effects, interactions) as well as compliance to maintain a good oral health. This latter aspect becomes fundamental if the OLP is solely or also localised on the gingival surfaces (i.e. desquamative gingivitis) as being susceptible to the common plaque-related gingivitis too.

Systemic therapy has been proposed in literature to control exacerbations of OLP [23], but topical application has been considered as the most suitable and effective as well as the easiest to practice and, hence, the first line of treatment [24].

In general, topical administration consists in a direct application on the lesions of natural substances/drugs or in use of a device (e.g. excimer laser), it has been found almost to be successful in oral soft tissue diseases, thanks to the easy accessibility of the mouth. However, it is important to underline that until today only few topical formulations are specific for oral mucosa diseases, whilst most of them are adopted from dermatology with all the limitations associated to this; let us consider that the structural and functional features of the oral mucosa, above all in terms of permeability, are 


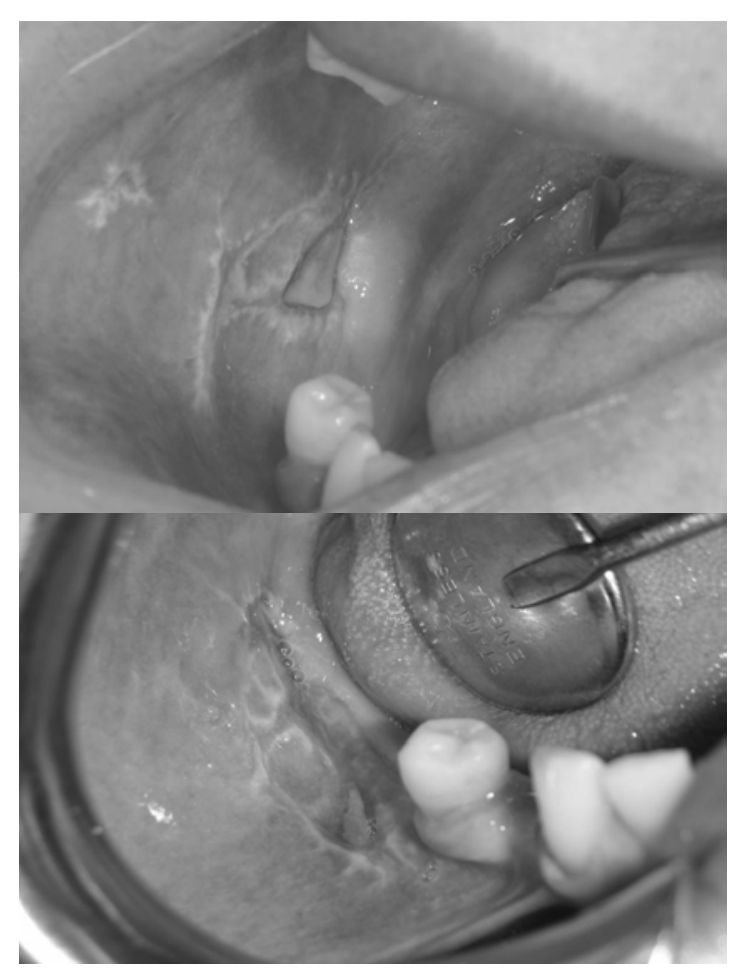

Fig. (1). Bullous OLP: before and after treatment with topical clobetasol proprionate.
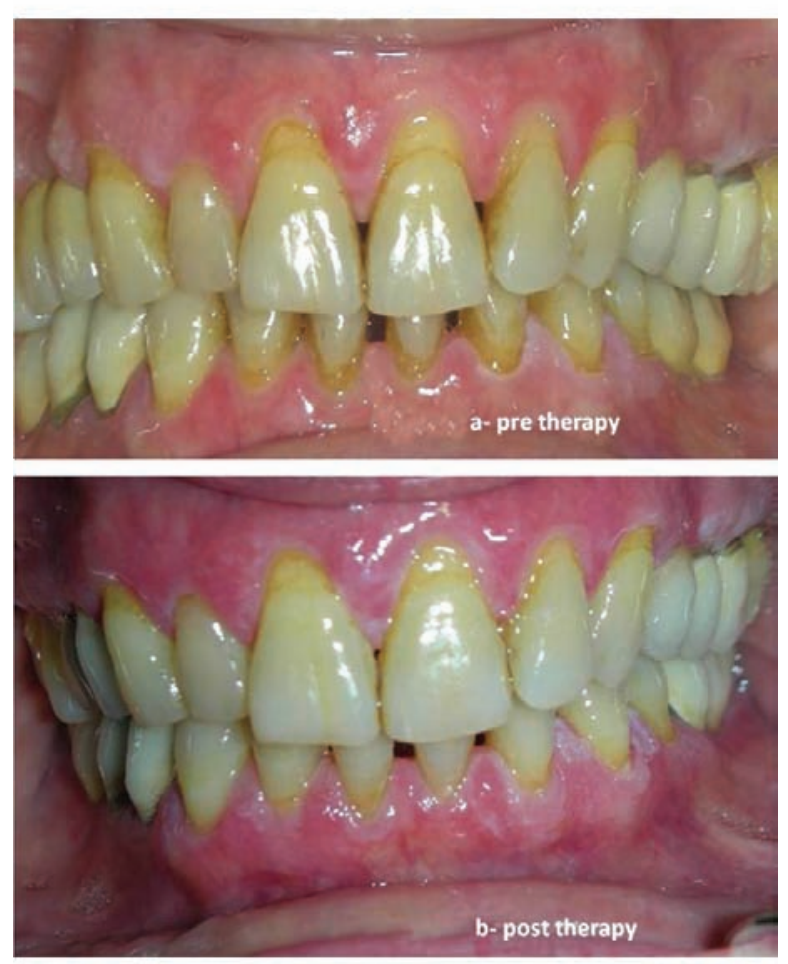

Fig. (2). Desquamative gingivitis hystologically confirmed as OLP: before and after treatment with topical clobetasol proprionate.

different to those of the skin. The presence of salivary flow that constantly wets the oral surfaces, in association with the swallowing, chewing and phonation, acts washing away most of the drug from the site of application resulting in a short retention time of the dosage forms and consequently in a low therapeutic efficacy. In addition, the presence of salivary enzymes could degrade the drug molecules and the bioadhesive polymers from the dosage forms. However, the oral mucosal surface is coated by a thin layer of mucus, that on one hand limits functions of permeability barrier, but on the other hand is one of the most important structures for the bioadhesion of the drug delivery systems [25]. To resolve all the limitations linked to the oral environment, the research today concentrates on the development of new bioadhesive formulations of the dosage forms to allow an efficient oral local drug delivery. In order to allow a longer residence time of the drug on the application site and consequently to a better bioavailability and therapeutic efficacy, the dosage forms should have in their formulation: a) mucoadhesive polymers (in order to provide an intimate and prolonged contact with the absorption site); b) chemical penetration enhancers (in order to improve drug permeation across oral mucosa or drug penetration into the deepest layers of the epithelium); and c) enzymatic inhibitors, in order to eventually protect drugs from the enzymatic degradation.

As stated above, oral local drug delivery consists in a more efficient drug-delivery approach than the systemic delivery one for the treatment of oral mucosal conditions. The reasons to prefer a local drug delivery to treat the oral soft tissue diseases are linked to their high frequency, their chronic status (requiring chronic treatment regimens) and above all to their excellent response to topical drugs.

Topical drug application avoids the need for ingestion and systemic distribution of drugs, provides a more targeted delivery as smaller amounts of drug can be easily targeted to the not healthy site, thereby reducing side effects.

The main disadvantages of local oral drug delivery are related to small surface area of the oral mucosa, to the limited exposure times [26] and to the bad taste sensation (especially during longterm treatments). Hence, local drug delivery should be considered a delivery route appropriate for drugs exhibiting high therapeutic potency as relatively small quantities of drugs can be delivered.

Mucoadhesive systems for the oral local drug delivery include: adhesive tablets, adhesive patches, adhesive films or pellicles, adhesive semi-solid systems (gels, ointments), adhesive liquid systems (sprays, mouthwahses).

As regards the application in the field of OLP, mucoadhesive tablets, [11, 27-29], bioadhesive gels [30], lipid microspheres [31] and oral rinses [32] are the most studied mucoadhesive dosage form for oral topical drug delivery.

When a mucosal condition as OLP has an immunologic pathogenesis, treatment with topical immunomodulators may be appropriate [27, 33-35]. The most prescribed topical drugs (Table 2) are corticosteroids for their anti inflammatory and immunosuppressant properties [36], with the use of two to three times a day, while further systemic dosage forms are reserved only in some particular and refractory cases.

In a recent review [32], Thongprasom et colleagues assessed the effectiveness and safety of any form of therapy for symptomatic OLP, concluding that although topical steroids are considered to be first line treatment, but there is no evidence that one steroid is any more successful than another. Furthermore, they continue to validate aloe vera as able to reduce the pain of OLP and improve the clinical signs of disease compared to placebo as well as cyclosporine, while there is no evidence that other calcineurin inhibitors reduce pain compared to either steroids or placebo. From the 28 trials included in this systematic review, the extensive collection of interventions compared means there is insufficient evidence to support the effectiveness of any treatment as being superior.

Besides, recently it has been considered as useful topical calcineurin inhibitors in OLP management as second-line treatment $[33,37]$. 
Table 1. Algorithm of management of OLP lesions (modified from Carrozzo M et al, 2009 and Lodi G et al, 2005)

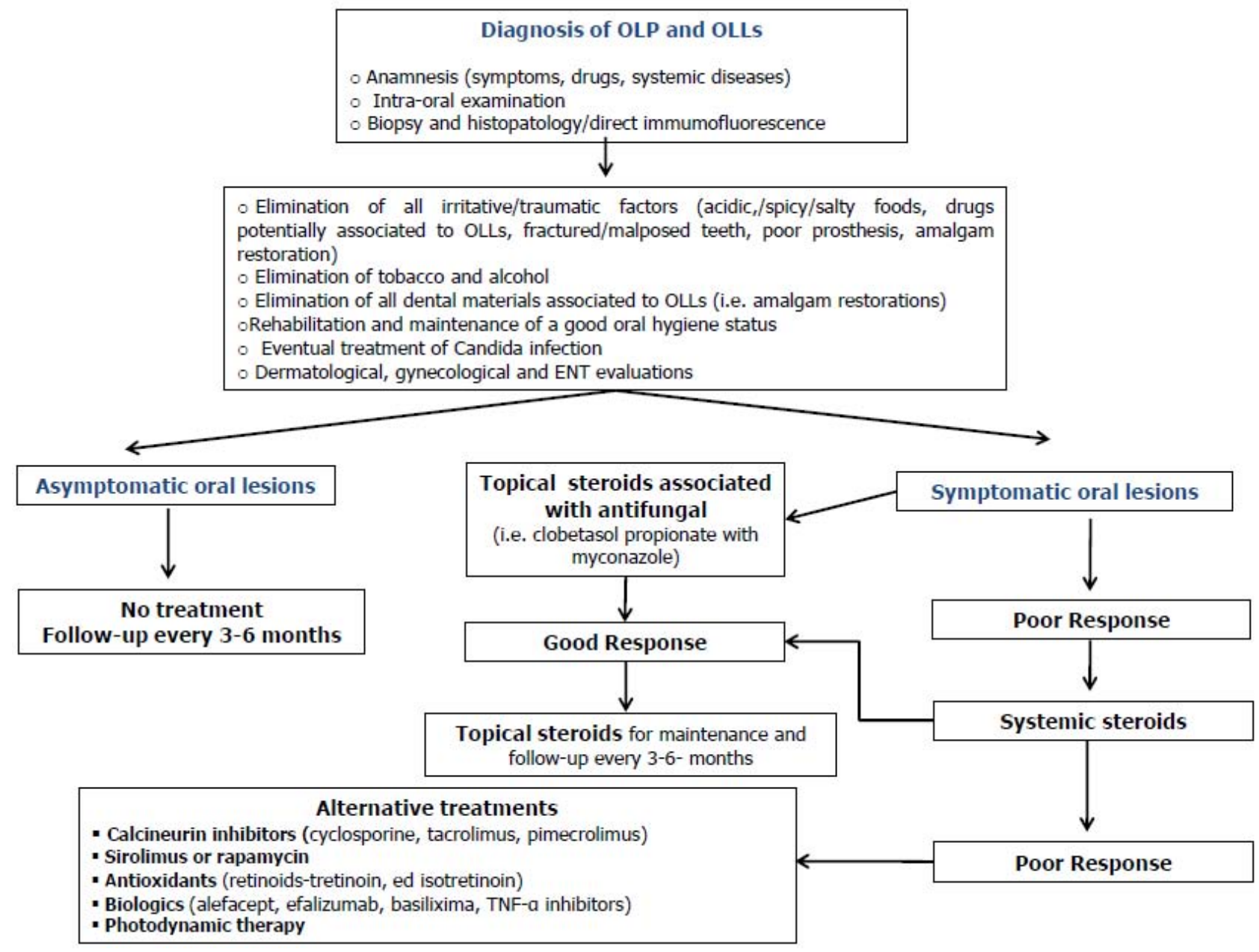

The aim of this paper is to review the available literature regarding any topical substances or applications to treat OLP, this in the view to help any specialist involved in managing this treatment and the respective pros and cons.

\section{Steroids}

A variety of synthetic glucocorticoids has been projected for therapeutic use and each substance is characterized by glucocorticoid potency, duration of effect, and overlapping mineralocorticoid potency varies. Hydrocortisone (pharmaceutical preparations of cortisol) is the standard of comparison for glucocorticoid potency. Topical steroids are categorized in potency class according to relative anti-inflammatory activity, but the action could differ significantly depending upon the vehicle, the site of application, the disease or the patient. Oral potency may be less than parenteral potency because significant amounts (up to $50 \%$ in some cases) may not be absorbed from the intestine. Topical corticosteroids as triamcinolone acetonide $0.1 \%$, fluocinolone acetonide $0.1 \%$ and fluocinonide $0.05 \%$ and clobetasol proprionate $0.05 \%$ are principally investigated and used for treatment of OLP.

In particular, clobetasol -17-propionate, a synthetic corticosteroid analogue of prednisolone, has met a great consensus; it is the most potent (highest potency class) topical steroid [38], with a complete decrease rate of OLP lesions from $47 \%$ to $75 \%$ [39].

The drug is very effective as a vasoconstrictor, an antiinflammatory and antiproliferative agent; moreover when topically administered, it allows good management of diseases reducing sys- temic side-effects for the patient. Prolonged contact with the oral mucosa should be avoided, since this may damage mucosal barriers and induce local immunosuppression, predisposing to oropharyngeal candidosis, one of the most common side-effects of topical corticosteroid therapy $[40,41]$. For this reason, a concomitant administration of topical antifungal drugs is often proposed.

Clobetasol propionate ointment $(0.05 \%)$ is demonstrated to be more successful than triamcinolone acetonide and fluocinonide in clinical trials [42].

Steroid mouthwashes (betametasone $500 \mu \mathrm{g}$ dissolved in $20 \mathrm{~mL}$ of water or clobetasol in acqueous solution) are used in cases of widespread OLP, even though systemic effects could be secondary.

Sometimes, topical application could be inadequate and not effective due to the aqueous solution, hence some adhesive denture pastes [16] and ointments [30] have been proposed over the last decade.

The extent of absorption by a topical corticosteroid, including clobetasol propionate, is determined by the vehicle or others factors, related to the applied dosage form as well as the integrity of the buccal mucosal barrier. Campisi et al. [26] evaluated the efficacy and compliance of a new delivery approach, loaded lipid microspheres, in an observer-blinded randomized clinical trial (RCT) of patients with OLP. Furthermore, Cilurzo et al [43] recently reported the excellent adhesion of tablets containing $24 \mu \mathrm{g}-$ clobetasol 3 times/day and confirmed the effectiveness in absence of systemic side effects. 
Table 2. Topical Treatments Proposed for OLP

\begin{tabular}{|c|c|c|}
\hline STEROIDS & ANTIOXIDANTS & OTHERS \\
\hline Triamcinolone acetonide $0.1 \%$ & Retinoids & Tetracycline $^{\mathrm{h}}$ \\
\hline Fluocinolone acetonide $0.1 \%$ & $>$ Tretinoin $^{\mathrm{a}}$ & Sirolimus or rapamycin ${ }^{\mathrm{h}}$ \\
\hline Fluocinonide $0.05 \%{ }^{\mathrm{a}}$ & Tarazotene $e^{\mathrm{a}, \mathrm{i}}$ & Hyaluronic acid $^{\mathrm{ajj}}$ \\
\hline Clobetasol propionate $^{\mathrm{a}, \mathrm{b}}$ & $>$ Isotretinoin $^{\mathrm{a}}$ & Aloe vera ${ }^{a, k}$ \\
\hline Fluticasone propionate $^{c}$ & $>$ Fenretinide & Herbal medicines \\
\hline Betamethasone sodium phosphate ${ }^{c}$ & & Laser Therapy \\
\hline \multirow[t]{2}{*}{ Mometasone furoate ${ }^{\mathrm{d}}$} & BIOLOGICS & $>$ LLLT $^{1}$ \\
\hline & Alefacept & Laser evaporation ${ }^{1}$ \\
\hline CALCINEURIN INHIBITORS & Efalizumab & Photo Dynamic Therapy ${ }^{1}$ \\
\hline Cyclosporine $\mathrm{e}^{\mathrm{a}, \mathrm{e}}$ & Basilixima & Extracorporeal photochemotherapy ${ }^{1}$ \\
\hline Pimecrolimus ${ }^{\mathrm{a}, \mathrm{f}}$ & TNF- $\alpha$ inhibitors & \\
\hline \multirow[t]{5}{*}{ Tacrolimus $^{\mathrm{a}, \mathrm{g}}$} & Etanercept & \\
\hline & Infliximab & \\
\hline & Adalimumab & \\
\hline & BCG-PSN $^{\mathrm{a}}$ & \\
\hline & Rituximab & \\
\hline
\end{tabular}

DESCRIPTION OF EFFICACY - ${ }^{\mathrm{a}}$ Efficacy confirmed by controlled-clinical trial; ${ }^{\mathrm{b}}$ more efficacy than triamcinolone acetonide and fluocinonide and few side effects; ${ }^{\mathrm{c}}$ effective in the short-term clinical management; ${ }^{\mathrm{d}}$ pilot phase II clinical trial; ${ }^{\mathrm{e}}$ reviews suggest a non well clinical response; ${ }^{\mathrm{f}}$ a medium/long term follow up has been reported only from cases report studies, more researches and comparisons with other treatments are needed; ${ }^{\mathrm{g}}$ represent a likely alternative to topical steroids; ${ }^{\mathrm{h}}$ only few reports published; ${ }^{\mathrm{i}}$ transient burning sensation and dysgeusia as side effects; ${ }^{\mathrm{T}}$ efficacy for up to $4 \mathrm{~h}$ after administration, it could be used as maintenance therapy of OLP, alternating it with topical corticosteroids; ${ }^{\mathrm{k}}$ efficacy on pain and burning sensation score, size and clinical characteristics of the lesions; 'only case series

In a randomized cross over study, Hegarty et al [44] investigated the acceptability and efficacy of topical fluticasone propionate spray ( $50 \mu \mathrm{g}$ two dose unit sprays) and betamethasone sodium phosphate mouthrinse (500 $\mu \mathrm{g} 4$ times daily), both administered for a period of 6 weeks, on the signs and symptoms of 44 OLP patients, evaluating also benefits of patient quality of life. Both substances caused a statistically significant reduction in painful symptoms, and improvement in quality of life. There was no significant difference between the two corticosteroids in their efficacy in reducing painful symptoms (measured by the VAS) or in their effect on patient quality of life. Fluticasone propionate was statistically significantly better than betamethasone sodium phosphate in reducing lesion surface area. Both are effective in the short-term clinical management of symptomatic OLP and fluticasone propionate is more suitable to patients than betamethasone sodium phosphate since it is in the spray form.

The efficacy of mometasone furoate microemulsion upon the symptoms and signs of OLP has been determined by a pilot phase II clinical trial [45]. In 49 subjects with erosive-ulcerative OLP, of $0.1 \%$ mometasone furoate microemulsion mouthwash was prescribed for three times a day over 30 days and pain, erythema and ulceration were assessed after 15 and 30 days of treatment. Mometasone caused a statistically significant reduction in pain, in clinical aspect and no one of these patients showed severe adverse effects, demonstrating that it is a safe and effective therapy in the treatment of symptomatic erosive-ulcerative OLP.

Efficacy of a triamcinolone acetonide, in two forms, has been evaluated in 20 OLP patients instructed to use a mouthwash $(\mathrm{n}=$ 11) or a commercially available triamcinolone acetonide paste $(\mathrm{n}=$ 9). The mouthwash was well accepted by patients and ten out of 11 patients treated for four weeks reported a positive response, and a complete response in signs and symptoms occurred in 4 and 5 of 11 patients, respectively. No significant difference in clinical improvement was observed between two formulations in OLP [46].

Another study [47] aimed to evaluate the efficiency and safety of triamcinolone acetonide by means injection in ulcerative OLP. Forty-five patients received $0.5 \mathrm{ml}$ triamcinolone acetonide (40 $\mathrm{mg} / \mathrm{ml}$ ) on lesions, after registration of visual analogue scale score and lesion areas at the time of injection and 1-week interval. After 2 weeks, if the treated ulceration reduced $<81 \%$ in size, a second injection was given. Patients revealed rapid relief of signs and symptoms, while the control group showed minimal decrease, since intralesional injection of triamcinolone acetonide in ulcerative OLP is effective and safe to achieve lesion and pain regression.

Of note, prolonged employ of these drugs could determine tachyphylaxis: for this reason, initially it is useful to prescribe a very potent steroid (e.g. clobetasol) and then, for maintenance therapy, a moderately potent steroid (e.g. triamcinolone) [48].

\section{Calcineurin Inhibitors (Cyclosporina, Pimecrolimus, Tac- rolimus)}

They are microbially derived immunosuppressive agents bind to cytoplasmic receptors of $\mathrm{T}$ lymphocytes, determining complexes that inhibit calcineurin and leading to suppression of cytokines transcription and production. Calcineurin-inhibitors as cyclosporine, tacrolimus, and pimecrolimus are of clinical benefit in oral immunomediated disease regimens.

Firstly, topical application of cyclosporine was reported by Eisen $[10,49-52]$ who compared it with placebo, showing an enhance- 
ment of clinical signs (e.g. erythema, erosion) and soreness in a small group received cyclospsorine. Different studies (RCT, open trails, case reports) [53] have investigated the efficacy of cyclosporine (prepared in a concentration of $15-100 \mathrm{mg} / \mathrm{ml}$ and administrated 3 times/day for 6-8 weeks) in OLP management. In a clinico-experimental evaluation [54] the Authors assessed topical therapy of OLP with cyclosporine by bioadhesive gel formula (carbosymethylcellulose) supporting adhesion for 2 hours. The regimen was carried out on 6 patients for 8 weeks with a low dose (48 $\mathrm{mg} /$ day) and showed remarkable results with a reduction ranging from 50 to $80 \%$ of oral lesions. On the contrary, other studies considered this result as not consistent, reporting a scarce help of cyclosporine application. Sieg et al. [55] have conducted a RCT determining that triamcinolone acetonide was better than cyclosporine in symptomatic OLP as well as Voute's group in an open trial [56]. Also with respect to the use of sulodexid (via intramuscular- 600 units before and by oral 250 units 2 times/day) during an open trial, cyclosporine rinse $(100 \mathrm{mg} / \mathrm{ml} 3$ times/day for 3 minutes $)$ has shown less rapid healing [11]. In a RCT [57], though clinically it has been observed a better improvement in patients undergone to steroid respect to cyclosporine group, the enhancement of pain is similar when patients applied steroids or cyclosporine. In a case series study $[13,58]$ it has been evaluated cyclosporine in patients with desquamative gingivitis: six patients used cyclosporine solution $(100 \mathrm{mg} / \mathrm{ml})$ to rinse one time for 5 minutes every day. In five patients it has been observed a complete resolution of gingival soreness within 2-4 weeks, while there were persistent lesions on dorsum of the tongue and gingival; hence, no patient had total healing of involved areas. Reviews on different topical and systemic regimens [59] have confirmed a not well response after administration of cyclosporine, with a lack of strong evidence of efficacy.

Pimecrolimus $1 \%$ cream has been tested in 20 patients for 2 months showing a statistically high significant improvement of lesions $(87 \%)$ and a significant decrease of VAS (93\%) and concluding that it appears to be a promising alternative treatment for OLP [18]. In a prospective randomized double-blind vehiclecontrolled study [60] it has been confirmed that pimecrolimus cream (1\%) could be helpful in patients with erosive OLP when it is applied twice daily for one month, determining healing (lesions and symptoms) in seven of ten patients versus an improvement only in two patients of the other group. When pimecrolimus was applied for further 30 days, a resolution has been observed in three subjects who do not respond to pimecrolimus the first month. Moreover, in a RCT pimecrolimus $1 \%$ cream, when applied for 2 months, did not confirm a better effectiveness of OLP symptoms and signs respect to topical triamcinolone acetonide [61] but both groups showed enhancement of all variables with no significant differences. Passeron et al [62] observed an improvement of erosive OLP when patients are treated with pimecrolimus $1 \%$ cream during the first phase but also had relapsed after cessation of therapy. Furthermore, blood levels of pimecrolimus remained above threshold with a mean value of $2.84 \mathrm{ng} / \mathrm{ml}$. Only a transient burning sense is reported by some patients. In a 6-week randomized, double-blind, vehicle-controlled phase followed by a 6-week open-label phase, 21 patients with erosive OLP were randomized and treated with moreover pimecrolimus $1 \%$ cream or vehicle cream to evaluate the effectiveness and safety of pimecrolimus. Pimecrolimus $1 \%$ cream was superior to vehicle in reducing pain, erythema, decreasing erosion size, and improving overall severity of disease when compared with vehicle treatment $[19,63]$. To our knowledge, a medium/long term follow up has been reported only from cases report studies [64-66] with a confirmation of pimecrolimus as an efficient potential treatment for OLP, although more research is needed to evaluate the efficacy of pimecrolimus and to study potential, as well as to establish its true role in comparison with other treatments for OLP.
Regarding tacrolimus, its efficacy has been evaluated by several open-label prospective studies [15, 16], randomized trials [67, 68], retrospective studies $[69,70]$, case series $[71,72]$ and case reports [70]. Topical tacrolimus, in two concentrations $0,03 \%$ and $0,1 \%$, is competent to obtain relief of pain, even if the first one does not warrant a complete healing, whereas its cessation determines recurrences within 1 or 2 weeks [13]. Since minimal side effects are reported [15] $(<25 \%$ of treated individuals), the use of tacrolimus could be suggested as a first line treatment in steroid- recalcitrant lesions, in susceptible patients for candidiasis and for other immunosuppressive-adverse effects. Tacrolimus could be considered as notably superior to topical corticosteroids for the treatment of OLP, but available data should be warily assessed since dissimilar preparations and concentrations of tacrolimus are used.

In a RCT Corrocher et colleagues [73] treated 32 OLP patients with tacrolimus $(0,1 \%)$ or clobetasol $(0,05 \%)$ ointment (four times/day for four weeks). Generally, symptoms ameliorated in both group after treatments, thought scores (e.g. pain, burning, lesions size) were lower in tacrolimus group compared to clobetasol one, even if in a first phase nine subjects felt a worsening that has been resolved after a week. In either group no side effects have been described.

In another study [65], it was compared the efficacy of topical tacrolimus ointment with triamcinolone acetonide ointment in patients with oral lichen planus. In a group of 20 patients, they were treated with topical tacrolimus $0.1 \%$ ointment 4 times daily, and in another group of 20 , they applied triamcinolone acetonide $0.1 \%$ in ointment 4 times daily; both groups used ointments for 6 weeks. In the first group, 6 patients revealed healing, 12 improvement and 2 showed no improvement. In the second group, 2 patients healed, 7 improved and 11 remained without improvement. Temporary burning or stinging at the site of application was the most common reported side-effect in both groups. Unluckily, in 13 of the first group and in 7 of the second group, who had initially shown an improvement or healed, oral lesions reappeared after 3-9 weeks of ending of treatment. A better initial therapeutic response of topical tacrolimus $0.1 \%$ ointment was determined respect to use of triamcinolone acetonide $0.1 \%$ ointment.

In a prospective study [74] 10 patients affected by refractory OLP used tacrolimus mouthwash $(0,01 \mathrm{mg} / 100 \mathrm{ml}$ in distilled water): seven of eight subjects that completed the study, improved clinically and in symptoms in six months. After the end of therapy, OLP relapsed and they were treated with steroids to which they responded well. Authors concluded that tacrolimus rinse in low concentration could relieve the symptoms effectively.

In an open clinical trial [13] it has been appraised the clinical efficacy and safety profile of tacrolimus powder in orabase $0.1 \%$ in 7 patients with OLP and 3 lichenoid lesions.

Before initiating tacrolimus regimen, all patients received a 1 week treatment of fluconazole, the most symptomatic site were treated, three times a day for two weeks; after two weeks, ameliorated of signs and symptoms was achieved in most patients. Authors concluded that tacrolimus powder in orabase $0.1 \%$ appeared to have a relatively safe profile, and represents a likely alternative to topical steroids in the treatment of OLP and lichenoid lesions, especially in those at risk for oral candidosis.

There is no objective evidence supporting an increased risk of oral malignancy in association with use of topical tacrolimus and pimecrolimus [75, 76], although some authors [77-85] reported two cases of carcinoma in OLP patient treated with topical tacrolimus for long term.

\section{Tetracycline}

They are widely prescribed for infections supported by a variety of gram positive bacterial microorganisms and by others as mycoplasms, chlamydiae, rickettsiae. The topical efficacy of tetracy- 
clines in recurrent aphthouts stomatitis and white sponge nevus has been assessed and published [86].

Regarding use of tetracyclines in OLP, in the literature there is a report of significant amelioration in a patient after one week of rinses of tetracycline solution $(0,25 \%)$ for $2-4 \mathrm{~min}$, three times per day, the resolution of erosive OLP with healing has been observed after six weeks, although white lesions continued [87].

\section{Sirolimus}

Also known as rapamycin, it is an immunosuppressant drug used to prevent rejection in organ transplantation. Sirolimus was originally developed as an antifungal agent but this use was abandoned when it was discovered to have potent immunosuppressive and antiproliferative properties. It has been studied for OLP topical management in a small study [88] rapamycin solution $(1 \mathrm{mg} / \mathrm{ml})$ was applied for three months twice a day in erosive OLP lesions in seven patients; although a patients gave up the study since a burning sensation for the application of sirolimus, after 3 months four patients had total resolution and two a partial remission.

\section{Antioxidants}

The oxidative stress plays an important role in the pathophysiology of OLP increasing the release of cytokines and free radicals (ROS) by damaged keratinocytes [89].

Thirthy years ago, Gunther [90] described the use of retinoids (tretinoin) in the treatment of OLP: the retinoids as physiological metabolites of retinol (vitamin A) have been used as antikeratinizing factor to regulate the synthesis of keratins through a specific action on the sulfhydryl radicals. These metabolites have antioxidant and anti-inflammatory properties and are involved in many physiological processes, particularly with regard to cellular differentiation [91].

The efficacy of topical isotretinoin gel $0.1 \%$ has been also assessed by Piattelli et al. in a placebo-controlled study. Ten patients with OLP were treated for 4 months with $0.1 \%$ isotretinoin gel and another ten patients with placebo. After treatment with isotretinoin, patients showed a complete or partial response, whereas, in the control group, no improvement of lesions has been observed. At the 3 -year follow-up complete healing was achieved in 9 of the 15 patients, whereas in 6 cases there was a recurrence of the lesion. Histologically and immunohistochemical analysis with antibodies against $\mathrm{Bcl}-2$ (protein implicated in apoptosis regulator process) and Ki-67 (nuclear protein associated with ribosomal RNA transcription) showed a reduction of apoptotic bodies accompanied by an increase of Bcl-2 and Ki- 67. The researchers concluded that the increase of these proteins could have contributed to the healing process. Differently from the systemic formulations, the topical dosage regimen would allow an impressive effect resulting in a higher concentration of the substance directly to the surface with few adverse effects [92].

In a double- blind placebo-controlled study, Petruzzi et al. treated 12 patients with an adhesive gel of tarazotene $(0,1 \%)$ applied twice a day on the lesional mucosal tissue for eight weeks. The authors concluded that topical gel of tarazotene seems to be more effective in test group versus the control one. Transient burning sensation and dysgeusia represented the minimal side effects observed [29].

\section{Hyaluronic Acid}

Hyaluronic acid (HA) is a glycosaminoglycan (GAG), with the major role in tissue healing: it is implicated in a range of activities including activation and moderation of the inflammatory responses, promoting cell proliferation, migration and angiogenesis, promoting re-epithelization, via proliferation of basal keratinocytes and reducing collagen disposition and scarring [29].
In a blinded parallel-group randomized clinical trial [93] the efficacy of a local HA preparation (0.2\%) was evaluated in 120 patients with erosive OLP. All subject were instructed to apply on the lesions 4-5 times daily for a total of 28 days. In addition, 5 and 60 minutes after each application the patients recorded scores of oral function and soreness on a clinical diary. In patients treated with HA, a decrease of pain for up to 4 hours post application and a reduction of ulcerative areas were registered. Furthermore, no significant differences between the two groups in relationship to oral function was found. The authors suggest that regular and very frequent applications of HA could be taken into account in order to obtain a more lasting action [94].

Cirillo's data $[95,96]$ demonstrate that keratinocytes represent a previously underestimated target for HA action in wound healing and serve as a peripheral neuroendocrine system that may play a role in nociception. HA preparations induce transcriptional changes in keratinocytes and stimulate wound closure. Furthermore, the addition of synthetic aminoacids to sodium hyaluronate (Aminogam $($ ) induces a distinct transcriptional profile and potentially promotes analgesia via secretion of endorphin in keratinocytes. The same compounds, in a spray dosage form, have been successfully used for the treatment of radio/chemotherapy-induced oral mucositis, with a significantly and positive impact on pain and lesion amelioration [97]. Although no controlled randomized clinical trial exists, at this regard HA being a natural compound without side effects could be used as maintenance therapy of OLP.

\section{Aloe Vera}

A double blind, placebo-controlled trial was designed by Choonhakarn et al. [98] in fifty-four patients assigned in two groups of 27 OLP subjects. The patients were instructed for application of Aloe vera (AV) twice daily for a total of 8 weeks. Improvement without complete healing of ulcerative lesions was observed in 22 of patients treated with AV, while one of 27 patients who received placebo had a similar response. Poor feedback was achieved in 2 AV subjects compared with 13 placebo patients. An absence of improvement in one AV patient and in 13 placebo patients have been registered. Disappearance of lesions was observed in 2 patients treated with AV, while none of the placebo group had a complete remission. Only in seventeen patients treated with AV, alleviation of the clinical symptoms were observed versus two placebo patients. Burning pain completely disappeared in nine patients treated with AV (33\%) and in one treated with placebo [99].

Also Salazar-Sánchez et al [100] have confirmed that the topical application of AV could improve the total quality of life score in patients with OLP.

In a randomized double-blinded clinical trial, Mansourian et al. [101] aimed to compare the therapeutic effects of aloe vera (AV) mouthwash with triamcinolone acetonide $0.1 \%$ (TA) on OLP. They observed that baseline characteristics, including pain and burning sensation score, size and clinical characteristics of the lesions according to Thongprasom index, were reduced in the 2 treatment groups, concluding that $\mathrm{AV}$ mouthwash is an effective substitute for TA in the treatment of OLP.

\section{Herbal Medicines}

Herbology is one of the most important treatment modalities for oral diseases utilized by Traditional Chinese Medicine (TCM) [101]. Each herbal prescription is a cocktail of several herbs. Even though TCM has studied for 2000 years for the treatment of different diseases, it is not completely understood and accepted by the clinicians outside China, especially since it is not based on an evidence-based system, but experience-based. The Chinese pharmacopoeia lists over 6000 medicinal substances and their properties. Among these substances around 600 herbs are commonly used today. Among these Liuwei Dihuang, Tripterygium wilfordii, Composite Taixian tablet, and Zengshengping are commonly used in the 
treatment of a wide spectrum of autoimmune and inflammatory diseases, demonstrating efficacy in treating oral diseases including oral lichen planus, but sometimes lacking standard criteria of posttreatment assessment and laboratory evidence. Zheng [102] reported that in 1997 Xian investigated the concomitant administration of Liuwei Dihuang and retinoic acid cream in 43 patients with OLP with more effective results than retinoic acid cream alone, particularly for treating the patients with a history of OLP less than 3 years.

\section{Laser Therapy}

Laser consists of an active medium hit by emitted energy from an external source (pumping system), that absorbs photons and transfers electrons from one energy level to a higher (inversion population). The active medium is a structure called "optical resonator" that allows photons emitted spontaneously to trigger the mechanism of stimulation and to generate, after a threshold, the laser beam. The emitted photons will be characterized by frequency, amplitude and wavelength.

In relation to the active medium, there are several types of lasers used in oral medicine: diode laser, excimer laser, low-level laser therapy (LLLT), $\mathrm{CO}_{2}$ laser.

Aghahosseini et al. [103] experienced the treatment of 26 lesions in 13 patients, evaluating the signs, symptoms and size of the lesions. A diode laser $\left(632 \mathrm{~nm}, 120 \mathrm{~J} / \mathrm{cm}^{2}\right)$ for 2 minutes was used. The analysed data showed a significant reduction in the size of lesions and a decrease of pain in 16 lesions.

Köllner et al. [104] used the excimer laser $308 \mathrm{~nm}-U V B$ for the intraoral treatment in eight patients with OLP. During treatment, the lesions were exposed to a variable number of application (from 9 to 32 ) and energy density per minute (from 75 to $175 \mathrm{~mJ} / \mathrm{cm}^{2}$ ). The results showed improvement in six patients, in particular complete remission was achieved in two cases, while one patient showed recurrence of the lesions after 4 weeks.

In an single center observational study, Trehan et al. [105] assessed the efficacy of excimer laser therapy (initial fluencies 100 $\mathrm{mJ} / \mathrm{cm}^{2}$, subsequently once a week $400 \mathrm{~mJ} / \mathrm{cm}^{2}$ ) in management of nine patients affected by OLP in a follow up ranged from 2 to 17 months. Low-dose treatment with the excimer 308-nm laser can be very effective in treating symptomatic and especially erosive OLP, an otherwise notoriously difficult-to-control disease.

On the other hand, not satisfactory results have been reported by Passeron [106] in four patients presenting erosive OLP. The authors evaluated the use of $308 \mathrm{~nm}$ excimer laser twice a week for 12 sessions. The lowest dose was $50 \mathrm{~mJ} / \mathrm{cm}^{2}$, increased by 50 $\mathrm{mJ} / \mathrm{cm}^{2}$ every two sessions up to $200 \mathrm{~mJ} / \mathrm{cm}^{2}$. Only one patient showed positive results.

The low-level laser therapy (LLLT) was described by Cafaro et $a l$, using $904 \mathrm{~nm} 4 \mathrm{~J} / \mathrm{cm}^{2}$ per minute $(10 \mathrm{kHz}, 50 \%$ duty cycle) with a distance of approximately $2 \mathrm{~mm}$ from the oral mucosa. All 13 patients reported a reduction in clinical signs as well as in pain [107]. A comparative pilot study of LLLT versus topical corticosteroids in the treatment of erosive-atrophic OLP demonstrated that LLLT is as effective as topical corticosteroid therapy without any adverse effects and it may be considered as an alternative treatment for erosive-atrophic OLP in the future [108].

The $\mathrm{CO}_{2}$ laser was another investigated laser system in oral medicine; Van der Hem et al. [109] exposed 39 superficial OLP lesions to $\mathrm{CO}_{2}$ laser evaporation $\left(1,5-2.0 \mathrm{Joule} / \mathrm{mm}^{2}\right)$. During a follow-up period of 1-18 years (mean 8 years) 24 lesions showed no more signs of oral dysfunction (pain, burning sensation). Fifteen lesions showed clinical recurrence, six of these caused pain after complete epithelialization. After retreatment with $\mathrm{CO}_{2}$ laser evaporation of these six lesions, no pain complaint was observed. In all patients, complete epithelialization after treatment completion and retreatment was observed in 3 weeks.

\section{Photo Dynamic Therapy and Extracorporeal photochemother- apy}

Photodynamic therapy (PDT) is a treatment that uses a substance, called photosensitizer or photosensitizing agent, and a particular type of light, such as a laser. PDT could be considered a safe alternative treatment in patients who do not respond to standard therapy or with contraindications to steroids. Methylene Blue (MB) is an optimal photosensitizer for PDT, it has a strong absorption at wavelengths longer than $620 \mathrm{~nm}$, and it may be a preferred choice for superficial lesions in skin and oral cavity.

Aghahosseini [110] investigated the effectiveness of photodynamic therapy with $\mathrm{MB}$ in two patients with oral lichen planus. The patients were instructed to gargle a 5\% methylene blue solution in water for 5 minutes $(0.05$ gr per $100 \mathrm{cc})$. After ten minutes the lesions were treated with light source (diode laser $632 \mathrm{~nm}, 100$ $\mathrm{J} / \mathrm{cm}^{2}$ ). They were followed up on sessions 3, 7, 15 days and 1 to 9 months after PDT. In two lesions, an improvement was achieved, and in two other lesions a complete remission was observed.

Extracorporeal photochemotherapy (ECP) or photopheresis, a type of PDT, is an emerging treatment in which a centrifugal apheresis machine is used to separate the patient's blood cells. The removed cell are treated with a photosensitizing compound (8methoxypsoralen), that was intercalated into DNA of treated cells making them more susceptible to apoptosis when exposed to ultraviolet A light. The cells are then re-infused to the patient.

ECP has been investigated by Kunte et al. [111] to assess their safety in 4 patients with erosive oral lichen planus reporting an improvement of symptoms and lesions in all subjects after seven to nine therapy cycles.

Twenty patients suffered from erosive lichen were enrolled in a study conducted by Guyot. ECP were performed twice weekly for 3 weeks while the following sessions were planned according to clinical improvement. The researchers monitored counts of peripheral blood CD $4+$, CD8 T- lymphocyte and subsets every 3 months. In all patients, clinical improvements were observed [112].

In another study ECP has been used successfully in the treatment of two patients with oral erosive and cortico-resistant lichen planus [113].

\section{Biologics}

The introduction of biologic therapies is a new challenge for the treatment of various immune-mediated disorder, in particular for unmanageable OLP. Biologics are intended by recombinant biotechnology to head off every step in the pathogenesis of immunoinflammatory diseases, representing a more effective and targeted approach. Structurally, biologics include receptor fusion proteins, monoclonal antibodies, and recombinant cytokines. Functionally, biologics can be divided into T-cell or cytokine modulators. As biotechnology agents directly andor indirectly target $\mathrm{T}$ cells, alefacept, efalizumab, basilixima, TNF- $\alpha$ inhibitors (i.e. etanercept, infliximab, adalimumab), polysaccharide nucleic acid fraction of bacillus Calmette-Guerin (BCG-PSN), and rituximab are used for treatments of OLP [114].

Xiong et al, [115] in a randomized control trial, have used immunologic active materials to regulate $\mathrm{T}$ cells (CD4 and CD8 cells) and subtypes of helper T cells (Th1 and Th2): topical injection (0.5 $\mathrm{ml}$ ) of Polysaccharide nucleic acid fraction of bacillus CalmetteGuerin (BCG-PSN) into the connective tissue below the erosive lesion from adjacent normal mucosa was compared with the intralesional injection $(10 \mathrm{mg})$ of Triamcinolone Acetonide (TA). The injection was administered weekly for 2 weeks. This study involved 56 patients ( 31 treated and 25 control) all affected with severe erosive OLP unresponsive to conventional therapies. There were no statistical differences between the two groups in the reduction of clinical signs (erosion), alleviation of pain and burning sensation, recurrence rate and occurrence of adverse reactions. These findings 
show that topical BCG-PSN are as effective as topical TA and might be useful as a therapeutic alternative for erosive OLP.

\section{DISCUSSION AND CONCLUSIONS}

The management of OLP is very difficult due to its chronic nature that requires a long-term treatment [116], and World Health Organisation considers OLP as "a generalized state associated with a significant increased risk of cancer". The cause of increased oral cancer risk in OLP patients is unknown but the highest rate of transformation regards especially the erosive and erythematic forms [36], that must be always treated.

In general, according to EAOM guidelines [117], OLP treatment is necessary only when it is symptomatic and regarding the therapeutic options, oral local drug delivery consists in a more efficient approach than systemic administration for the treatment of oral mucosal conditions. The reasons to prefer a local drug delivery to treat the oral soft tissue diseases are linked to their high frequency, their chronic status (requiring chronic treatment regimens) and above all to their excellent response to topical drugs.

There are several studies on molecule different from glucocorticosteroids, but not sufficient or statistically adequate to justify their evidence based use in OLP; a further criticism is the scarcity in literature of standardized evaluation parameters during the OLP management (e.g. lesions size, VAS scale).

Specifically, to assess the effectiveness and safety of any form of therapy for symptomatic OLP, Thongprasom and colleagues $[118,119]$ reviewed all randomised controlled clinical trials (RCTs) of OLP therapy with the Cochrane collaboration in which treatment with a placebo or between treatments or no intervention have been compared, concluding that there is no evidence that a specific treatment is more effective than another.

To date, the application of immunosuppressive drugs, such as glucocorticoid, appears to be the safest and most effective topical treatment available, even if there is much dispute about their longterm efficacy and benefits, and there is a wide scenario with several different molecules proposed, at least of auxiliary benefit.

It seems extremely interesting and novel the research investigating on endogenous steroidal system in keratinocytes $[118,119]$.

In fact, the bioavailability of circulating and/or endogenous hydrocortisone (cortisol) in epidermal cells is a key determinant in inflammatory disease. However, it is unknown whether epidermal cells can regulate tissue cortisol and whether they are capable of producing endogenous glucocorticoids [117, 118]. A further key observation is that keratinocytes responded to stimulation with ACTH by a significant increase in the de novo synthesis of cortisol. The same authors have recently reported that epidermal keratinocytes can be considered a new non-adrenal glucocorticoid- system endogenously implicated in regulation of cortisol: data showed that oral fibroblasts and keratinocytes were able to activate cortisone into the active form cortisol, and confirmed the capacity of synthesizing cortisol de novo following stimulation with ACTH; particularly, when the isoenzyme11b-Hydroxysteroid dehydrogenase (HSD) $1 / 2$ is present, it controls cortisol deactivation. Supplementary close examinations to control bioavailability of circulating cortisol in epidermal cells are awaited, and well-designed randomized controlled trials should be carried out to establish the accurate effectiveness of topical administration for the treatment of immunologically mediated oral mucosal disease as OLP.

\section{CONFLICT OF INTEREST}

The authors confirm that this article content has no conflicts of interest.

\section{ACKNOWLEDGEMENTS}

B.J. and C.D. contributed equally to this work.

\section{REFERENCES}

[1] Farhi D, Dupin N. Pathophysiology, etiologic factors, and clinical management of oral lichen planus, part I: facts and controversies. Clin Dermatol 2010; 28(1): 100-8.

[2] Eisenberg E. Oral lichen planus: A benign lesion. J Oral Maxillofac Surg 2000; 58(11): 1278-85

[3] Eisen D. The evaluation of cutaneous, genital, scalp, nail, esophageal, and ocular involvement in patients with oral lichen planus. Oral Surg Oral Med Oral Pathol Oral Radiol Endod 1999; 88(4): 431-6.

[4] Rogers RS 3rd, Eisen D. Erosive oral lichen planus with genital lesions: The vulvovaginal-gingival syndrome and the peno-gingival syndrome. Dermatol Clin 2003; 21(1): 91-8.

[5] Di Fede O, Belfiore P, Cabibi D, et al. Unexpectedly high frequency of genital involvement in women with clinical and histological features of oral lichen planus. Acta Derm Venereol 2006; 86(5): 433-8

[6] Scully C, Carrozzo M. Oral mucosal disease: Lichen planus. Br J Oral Maxillofac Surg 2008; 46(1): 15-21.

[7] Mollaoglu N. Oral lichen planus: A review. Br J Oral Maxillofac Surg 2000; 38(4): 370-7.

[8] Xue JL, Fan MW, Wang SZ, Chen XM, Li Y, Wang L. A clinical study of 674 patients with oral lichen planus in China. J Oral Pathol Med 2005; 34(8): 467-72.

[9] Scully C, Eisen D, Carrozzo M. Management of oral lichen planus. American Journal of Clinical Dermatology 2000; 1(5): 287-306.

[10] Yoke PC, Tin GB, Kim MJ, et al. A randomized controlled trial to compare steroid with cyclosporine for the topical treatment of oral lichen planus. Oral Surg Oral Med Oral Pathol Oral Radiol Endod 2006; 102(1): 47-55.

[11] Conrotto D, Carbone M, Carrozzo M, et al. Ciclosporin vs. clobetasol in the topical management of atrophic and erosive oral lichen planus: a double-blind, randomized controlled trial. Br J Dermatol 2006; 154(1): 139-45.

[12] Madan V, Griffiths CE. Systemic ciclosporin and tacrolimus in dermatology. Dermatol Ther 2007; 20(4): 239-50.

[13] Al Johani KA, Hegarty AM, Porter SR, Fedele S. Calcineurin inhibitors in oral medicine. J Am Acad Dermatol 2009; 61(5): 82940 .

[14] Xiong C, Li Q, Lin M, et al. The efficacy of topical intralesional BCG-PSN injection in the treatment of erosive oral lichen planus: a randomized controlled trial. J Oral Pathol Med 2009; 38(7): 551-8.

[15] Corrocher G, Di Lorenzo G, Martinelli N, et al. Comparative effect of tacrolimus $0.1 \%$ ointment and clobetasol $0.05 \%$ ointment in patients with oral lichen planus. J Clin Periodontol 2008; 35(3): 2449.

[16] Radfar L, Wild RC, Suresh L. A comparative treatment study of topical tacrolimus and clobetasol in oral lichen planus. Oral Surg Oral Med Oral Pathol Oral Radiol Endod 2008; 105(2): 187-93.

[17] Lopez-Jornet P, Camacho-Alonso F, Salazar-Sanchez N. Topical tacrolimus and pimecrolimus in the treatment of oral lichen planus: an update. J Oral Pathol Med 2010; 39(3): 201-5.

[18] Volz T, Caroli U, Ludtke H, et al. Pimecrolimus cream 1\% in erosive oral lichen planus--a prospective randomized double-blind vehicle-controlled study. Br J Dermatol 2008; 159(4): 936-41.

[19] Dissemond J. Pimecrolimus in an adhesive ointment is safe and effective in long-term treatment for oral lichen planus. J Eur Acad Dermatol Venereol 2008; 22(8): 1009-11.

[20] Boyce AE, Marshman G, Mills RA. Erosive mucosal lichen planus and secondary epiphora responding to systemic cyclosporin A treatment. Australas J Dermatol 2009; 50(3): 190-3.

[21] Riano Arguelles A, Martino Gorbea R, Iglesias Zamora ME, Garatea Crelgo J. Topic tacrolimus, alternative treatment for oral erosive lichen planus resistant to steroids: a case report. Med Oral Patol Oral Cir Bucal 2006; 11(6): E462-6.

[22] Chao TJ. Adalimumab in the management of cutaneous and oral lichen planus. Cutis 2009; 84(6): 325-8.

[23] Carbone M, Goss E, Carrozzo M, et al. Systemic and topical corticosteroid treatment of oral lichen planus: a comparative study with long-term follow-up. J Oral Pathol Med 2003; 32(6): 323-9.

[24] Khutoryanskiy VV. Advances in mucoadhesion and mucoadhesive polymers. Macromol Biosci 2011; 11(6): 748-64.

[25] Madhav NV, Shakya AK, Shakya P, Singh K. Orotransmucosal drug delivery systems: a review. J Control Release 2009; 140(1): 211 . 
[26] Cilurzo F, Gennari CG, Selmin F, et al. A new mucoadhesive dosage form for the management of oral lichen planus: formulation study and clinical study. Eur J Pharm Biopharm 2010; 76(3): 43742.

[27] Carbone M, Arduino PG, Carrozzo M, et al. Topical clobetasol in the treatment of atrophic-erosive oral lichen planus: a randomized controlled trial to compare two preparations with different concentrations. J Oral Pathol Med 2009; 38(2): 227-33.

[28] Sankar V, Hearnden V, Hull K, et al. Local drug delivery for oral mucosal diseases: challenges and opportunities. Oral Dis $2011 ; 17$ (Suppl 1): 73-84.

[29] Nolan A, Badminton J, Maguire J, Seymour RA. The efficacy of topical hyaluronic acid in the management of oral lichen planus. $\mathrm{J}$ Oral Pathol Med 2009; 38(3): 299-303.

[30] Campisi G, Giandalia G, De Caro V, Di Liberto C, Arico P, Giannola LI. A new delivery system of clobetasol-17-propionate (lipidloaded microspheres $0.025 \%$ ) compared with a conventional formulation (lipophilic ointment in a hydrophilic phase $0.025 \%$ ) in topical treatment of atrophic/erosive oral lichen planus. A Phase IV, randomized, observer-blinded, parallel group clinical trial. Br J Dermatol 2004; 150(5): 984-90.

[31] Rouxel AM, Le Toux G, Misery L. [Tacrolimus mouthwash as second-line treatment for erosive oral lichen planus]. Ann Dermatol Venereol 2010; 137(10): 648-9.

[32] Elad S, Epstein JB, Yarom N, Drucker S, Tzach R, von Bultzingslowen I. Topical immunomodulators for management of oral mucosal conditions, a systematic review; part I: calcineurin inhibitors. Expert Opin Emerg Drugs 2010; 15(4): 713-26.

[33] Thongprasom K, Dhanuthai K. Steriods in the treatment of lichen planus: a review. J Oral Sci 2008; 50(4): 377-85.

[34] Endo H, Rees TD, Kuyama K, Matsue M, Yamamoto H. Successful treatment using occlusive steroid therapy in patients with erosive lichen planus: a report on 2 cases. Quintessence Int 2008; 39(4): e162-72.

[35] Malhotra AK, Khaitan BK, Sethuraman G, Sharma VK. Betamethasone oral mini-pulse therapy compared with topical triamcinolone acetonide $(0.1 \%)$ paste in oral lichen planus: A randomized comparative study. J Am Acad Dermatol 2008; 58(4): 596602 .

[36] Thongprasom K, Carrozzo M, Furness S, Lodi G. Interventions for treating oral lichen planus. Cochrane Database Syst Rev 2011; (7): CD001168.

[37] Usatine RP, Tinitigan M. Diagnosis and treatment of lichen planus. Am Fam Physician 2011; 84(1): 53-60.

[38] Farhi D, Dupin N. Pathophysiology, etiologic factors, and clinical management of oral lichen planus, part I: facts and controversies. Clin Dermatol 2010; 28(1): 100-8.

[39] Ellepola AN, Samaranayake LP. Inhalational and topical steroids, and oral candidosis: a mini review. Oral Dis 2001; 7(4): 211-6.

[40] Lozada-Nur F, Miranda C, Maliksi R. Double-blind clinical trial of $0.05 \%$ clobetasol propionate (corrected from proprionate) ointment in orabase and $0.05 \%$ fluocinonide ointment in orabase in the treatment of patients with oral vesiculoerosive diseases. Oral Surg Oral Med Oral Pathol 1994; 77(6): 598-604.

[41] Carbone M, Conrotto D, Carrozzo M, Broccoletti R, Gandolfo S, Scully C. Topical corticosteroids in association with miconazole and chlorhexidine in the long-term management of atrophic-erosive oral lichen planus: a placebo-controlled and comparative study between clobetasol and fluocinonide. Oral Dis 1999; 5(1): 44-9.

[42] Lo Muzio L, della Valle A, Mignogna MD, et al. The treatment of oral aphthous ulceration or erosive lichen planus with topical clobetasol propionate in three preparations: a clinical and pilot study on 54 patients. J Oral Pathol Med 2001; 30(10): 611-7.

[43] Hegarty AM, Hodgson TA, Lewsey JD, Porter SR. Fluticasone propionate spray and betamethasone sodium phosphate mouthrinse: a randomized crossover study for the treatment of symptomatic oral lichen planus. J Am Acad Dermatol 2002; 47(2): 271-9.

[44] Aguirre JM, Bagan JV, Rodriguez C, et al. Efficacy of mometasone furoate microemulsion in the treatment of erosive-ulcerative oral lichen planus: pilot study. J Oral Pathol Med 2004; 33(7): 381-5.

[45] Ungphaiboon S, Nittayananta W, Vuddhakul V, et al. Formulation and efficacy of triamcinolone acetonide mouthwash for treating oral lichen planus. Am J Health Syst Pharm 2005; 62(5): 485-91.

[46] Xia J, Li C, Hong Y, Yang L, Huang Y, Cheng B. Short-term clinical evaluation of intralesional triamcinolone acetonide injection for ulcerative oral lichen planus. J Oral Pathol Med 2006; 35(6): $327-$ 31.

[47] Carrozzo M, Thorpe R. Oral lichen planus: a review. Minerva Stomatol 2009; 58(10): 519-37.

[48] Eisen D, Ellis CN, Duell EA, Griffiths CE, Voorhees JJ. Effect of topical cyclosporine rinse on oral lichen planus. A double-blind analysis. N Engl J Med 1990; 323(5): 290-4.

[49] Pacor ML, Biasi D, Urbani G, Lombardo G, Lunardi C. [The efficacy of cyclosporin for topical use in oral lichen planus]. Minerva Stomatol 1994; 43(4): 129-32.

[50] Frances C, Boisnic S, Etienne S, Szpirglas H. Effect of the local application of ciclosporine A on chronic erosive lichen planus of the oral cavity. Dermatologica 1988; 177(3): 194-5.

[51] Demitsu T, Sato T, Inoue T, Okada O, Kubota T. Corticosteroidresistant erosive oral lichen planus successfully treated with topical cyclosporine therapy. Int J Dermatol 2000; 39(1): 79-80.

[52] Chaklader M, Morris-Larkin C, Gulliver W, McGrath J. Cyclosporine in the management of esophageal lichen planus. Can $\mathrm{J}$ Gastroenterol 2009; 23(10): 686-8

[53] Gombos F, Cappello B, Gaeta GM, La Rotonda MI, Serpico R, Miro A. [Cyclosporin in a bioadhesive formulation in the therapy of oral erosive lichen planus. A clinico-experimental evaluation]. Minerva Stomatol 1992; 41(9): 385-9.

[54] Sieg P, Von Domarus H, Von Zitzewitz V, Iven H, Farber L. Topical cyclosporin in oral lichen planus: a controlled, randomized, prospective trial. Br J Dermatol 1995; 132(5): 790-4.

[55] Voute AB, Schulten EA, Langendijk PN, Nieboer C, van der Waal I. Cyclosporin $\mathrm{A}$ in an adhesive base for treatment of recalcitrant oral lichen planus. An open trial. Oral Surg Oral Med Oral Pathol 1994; 78(4): 437-41.

[56] Femiano F, Gombos F, Scully C. Oral erosive/ulcerative lichen planus: preliminary findings in an open trial of sulodexide compared with cyclosporine (ciclosporin) therapy. Int J Dermatol 2003; 42(4): 308-11.

[57] Porter SR, Scully C, Eveson JW. The efficacy of topical cyclosporin in the management of desquamative gingivitis due to lichen planus. Br J Dermatol 1993; 129(6): 753-5.

[58] Zakrzewska JM, Chan ES, Thornhill MH. A systematic review of placebo-controlled randomized clinical trials of treatments used in oral lichen planus. Br J Dermatol 2005; 153(2): 336-41.

[59] Ibrahim SS, Hazzaa HH. Topical pimecrolimus effect on Fas inducing apoptosis in oral lichen planus: a clinical immunohistochemical study. J Oral Pathol Med 2012; 41(4): 315-21.

[60] Gorouhi F, Solhpour A, Beitollahi JM, et al. Randomized trial of pimecrolimus cream versus triamcinolone acetonide paste in the treatment of oral lichen planus. J Am Acad Dermatol 2007; 57(5): 806-13.

[61] Passeron T, Lacour JP, Fontas E, Ortonne JP. Treatment of oral erosive lichen planus with $1 \%$ pimecrolimus cream: a double-blind, randomized, prospective trial with measurement of pimecrolimus levels in the blood. Arch Dermatol 2007; 143(4): 472-6.

[62] McCaughey C, Machan M, Bennett R, Zone JJ, Hull CM. Pimecrolimus $1 \%$ cream for oral erosive lichen planus: a 6-week randomized, double-blind, vehicle-controlled study with a 6-week open-label extension to assess efficacy and safety. J Eur Acad Dermatol Venereol 2011; 25(9): 1061-7.

[63] Esquivel-Pedraza L, Fernandez-Cuevas L, Ortiz-Pedroza G, ReyesGutierrez E, Orozco-Topete R. Treatment of oral lichen planus with topical pimecrolimus $1 \%$ cream. Br J Dermatol 2004; 150(4): 771-3.

[64] Kaliakatsou F, Hodgson TA, Lewsey JD, Hegarty AM, Murphy AG, Porter SR. Management of recalcitrant ulcerative oral lichen planus with topical tacrolimus. J Am Acad Dermatol 2002; 46(1): 35-41.

[65] Olivier V, Lacour JP, Mousnier A, Garraffo R, Monteil RA, Ortonne JP. Treatment of chronic erosive oral lichen planus with low concentrations of topical tacrolimus: an open prospective study. Arch Dermatol 2002; 138(10): 1335-8.

[66] Shichinohe R, Shibaki A, Nishie W, Tateishi Y, Shimizu H. Successful treatment of severe recalcitrant erosive oral lichen planus with topical tacrolimus. J Eur Acad Dermatol Venereol 2006; 20(1): 66-8.

[67] Thomson MA, Hamburger J, Stewart DG, Lewis HM. Treatment of erosive oral lichen planus with topical tacrolimus. J Dermatolog Treat 2004; 15(5): 308-14 
[68] Hodgson TA, Sahni N, Kaliakatsou F, Buchanan JA, Porter SR. Long-term efficacy and safety of topical tacrolimus in the management of ulcerative/erosive oral lichen planus. Eur J Dermatol 2003; 13(5): 466-70.

[69] Morrison L, Kratochvil FJ, 3rd, Gorman A. An open trial of topical tacrolimus for erosive oral lichen planus. J Am Acad Dermatol 2002; 47(4): 617-20.

[70] Rozycki TW, Rogers RS, 3rd, Pittelkow MR, et al. Topical tacrolimus in the treatment of symptomatic oral lichen planus: a series of 13 patients. J Am Acad Dermatol 2002; 46(1): 27-34.

[71] Lener EV, Brieva J, Schachter M, West LE, West DP, el-Azhary RA. Successful treatment of erosive lichen planus with topical tacrolimus. Arch Dermatol 2001; 137(4): 419-22.

[72] Shen JT, Pedvis-Leftick A. Mucosal staining after using topical tacrolimus to treat erosive oral lichen planus. J Am Acad Dermatol 2004; 50(2): 326.

[73] Laeijendecker R, Tank B, Dekker SK, Neumann HA. A comparison of treatment of oral lichen planus with topical tacrolimus and triamcinolone acetonide ointment. Acta Derm Venereol 2006; 86(3): 227-9.

[74] Lozada-Nur FI, Sroussi HY. Tacrolimus powder in Orabase $0.1 \%$ for the treatment of oral lichen planus and oral lichenoid lesions: an open clinical trial. Oral Surg Oral Med Oral Pathol Oral Radiol Endod 2006; 102(6): 744-9.

[75] Becker JC, Houben R, Vetter CS, Brocker EB. The carcinogenic potential of tacrolimus ointment beyond immune suppression: a hypothesis creating case report. BMC Cancer 2006; 6: 7.

[76] Mattsson U, Magnusson B, Jontell M. Squamous cell carcinoma in a patient with oral lichen planus treated with topical application of tacrolimus. Oral Surg Oral Med Oral Pathol Oral Radiol Endod 2010; 110(1): e19-25.

[77] Graykowski EA, Kingman A. Double-blind trial of tetracycline in recurrent aphthous ulceration. J Oral Pathol 1978; 7(6): 376-82.

[78] Gorsky M, Epstein J, Raviv A, Yaniv R, Truelove E. Topical minocycline for managing symptoms of recurrent aphthous stomatitis. Spec Care Dentist 2008; 28(1): 27-31.

[79] Gorsky M, Epstein J, Rabenstein S, Elishoov H, Yarom N. Topical minocycline and tetracycline rinses in treatment of recurrent aphthous stomatitis: a randomized cross-over study. Dermatol Online J 2007; 13(2): 1 .

[80] Guggenheimer J, Brightman VJ, Ship, II. Effect of chlortetracycline mouthrinses on the healing of recurrent aphthous ulcers: a double-blind controlled trial. J Oral Ther Pharmacol 1968; 4(5): 406-8.

[81] McDonagh AJ, Gawkrodger DJ, Walker AE. White sponge naevus successfully treated with topical tetracycline. Clin Exp Dermatol $1990 ; 15(2): 152-3$.

[82] Lim J, Ng SK. Oral tetracycline rinse improves symptoms of white sponge nevus. J Am Acad Dermatol 1992; 26(6): 1003-5.

[83] Otobe IF, de Sousa SO, Migliari DA, Matthews RW. Successful treatment with topical tetracycline of oral white sponge nevus occurring in a patient with systemic lupus erythematosus. Int J Dermatol 2006; 45(9): 1130-1.

[84] Otobe IF, de Sousa SO, Matthews RW, Migliari DA. White sponge naevus: improvement with tetracycline mouth rinse: report of four cases. Clin Exp Dermatol 2007; 32(6): 749-51.

[85] Becker LR, Lutz C, Erbard H, Brocker EB, Hamm H. White sponge naevus successfully treated with tetracycline mouth rinse. Acta Derm Venereol 1997; 77(5): 413.

[86] Walchner M, Messer G, Salomon N, Plewig G, Rocken M. Topical tetracycline treatment of erosive oral lichen planus. Arch Dermatol 1999; 135(1): 92-3.

[87] Soria A, Agbo-Godeau S, Taieb A, Frances C. Treatment of refractory oral erosive lichen planus with topical rapamycin: 7 cases. Dermatology 2009; 218(1): 22-5.

[88] Sander CS, Cooper SM, Ali I, Dean D, Thiele JJ, Wojnarowska F. Decreased antioxidant enzyme expression and increased oxidative damage in erosive lichen planus of the vulva. BJOG 2005; 112(11): 1572-1575.

[89] Günther SH. Vitamin A acid in treatment of oral lichen planus. Arch Dermatol 1973; 107(2): 277.

[90] Bollag W. The development of retinoids in experimental and clinical oncology and dermatology. J Am Acad of Dermatol 1983; 9(5): 797-805.
[91] Piattelli A, Carinci F, Iezzi G, et al. Oral lichen planus treated with 13-cis-retinoic acid (isotretinoin): Effects on the apoptotic process. Clin Oral Investig 2007; 11(3): 283-8.

[92] Petruzzi M, De Benedittis M, Grassi R, Cassano N, Vena G, Serpico R. Oral lichen planus: A preliminary clinical study on treatment with tazarotene. Oral Dis 2002; 8(6): 291-5.

[93] Nolan A, Badminton J, Maguire J, Seymour RA. The efficacy of topical hyaluronic acid in the management of oral lichen planus. J Oral Pathol Med 2009; 38(3): 299-303.

[94] Cirillo N. Molecular basis of the selective efficacy of aminoacidenriched sodium hyaluronate on wound healing. In: XI National Meeting of the Italian Society of Oral Medicine and Pathology. First International Congress; Pugnochiuso (FG), Italy, 16-18 June 2011.

[95] Colella G, Cannavale R, Vicidomini A, Rinaldi G, Compilato D, Campisi G. Efficacy of a spray compound containing a pool of collagen precursor synthetic aminoacids (1-proline, l-leucine, 1-lysine and glycine) combined with sodium hyaluronate to manage chemo/radiotherapy-induced oral mucositis: preliminary data of an open trial. Int J Immunopathol Pharmacol 2010; 23(1): 143-51.

[96] Colella G, Compilato. D. Update sulle mucositi - protocolli sperimentali. In: XI National Meeting of the Italian Society of Oral Medicine and Pathology. First International Congress; Pugnochiuso (FG), Italy, 16-18 June 2011..

[97] Choonhakarn C, Busaracome P, Sripanidkulchai B, Sarakarn P. The efficacy of aloe vera gel in the treatment of oral lichen planus: a randomized controlled trial. Br J Dermatol 2008; 158(3): 573-7.

[98] Choonhakarn C, Busaracome P, Sripanidkulchai B, Sarakarn P. The efficacy of aloe vera gel in the treatment of oral lichen planus: A randomized controlled trial. Br J Dermatol 2008; 158(3): 573-7.

[99] Salazar-Sanchez N, Lopez-Jornet P, Camacho-Alonso F, SanchezSiles M. Efficacy of topical Aloe vera in patients with oral lichen planus: a randomized double-blind study. J Oral Pathol Med 2010; 39(10): 735-40.

[100] Mansourian A, Momen-Heravi F, Saheb-Jamee M, Esfehani M, Khalilzadeh O, Momen-Beitollahi J. Comparison of aloe vera mouthwash with triamcinolone acetonide $0.1 \%$ on oral lichen planus: a randomized double-blinded clinical trial. Am J Med Sci 2011; 342(6): 447-51.

[101] Zheng LW, Hua H, Cheung LK. Traditional Chinese medicine and oral diseases: today and tomorrow. Oral Dis 2011; 17(1): 7-12.

[102] Aghahosseini F, Arbabi-Kalati F, Fashtami LA, Djavid GE, Fateh M, Beitollahi JM. Methylene blue-mediated photodynamic therapy: A possible alternative treatment for oral lichen planus. Lasers Surg Med 2006; 38(1): 33-38.

[103] Köllner K, Wimmershoff M, Landthaler M, Hohenleutner U. Treatment of oral lichen planus with the 308-nm UVB excimer laser - Early preliminary results in eight patients. Lasers Surg Med 2003; 33(3): 158-60.

[104] Trehan M, Taylor CR. Low-Dose Excimer 308-nm Laser for the Treatment of Oral Lichen Planus. Arch Dermatol 2004; 140(4): 415-20.

[105] Passeron T, Zakaria W, Ostovari N, Mantoux F, Lacour JPH, Ortonne JP. Treatment of Erosive Oral Lichen Planus by the $308 \mathrm{~nm}$ Excimer Laser [2]. Lasers Surg Med 2004; 34(3): 205.

[106] Cafaro A, Albanese G, Arduino PG, et al. Effect of Low-Level laser irradiation on unresponsive oral lichen Planus: Early preliminary results in 13 patients. Photomed Laser Surg 2010; 28(Suppl. 2): S99-S103.

[107] Jajarm HH, Falaki F, Mahdavi O. A comparative pilot study of low intensity laser versus topical corticosteroids in the treatment of erosive-atrophic oral lichen planus. Photomed Laser Surg; 29(6): 4215 .

[108] van der Hem PS, Egges M, van der Wal JE, Roodenburg JLN. CO2 laser evaporation of oral lichen planus. Int J Oral Maxillofac Surg 2008; 37(7): 630-3.

[109] Aghahosseini F, Arbabi-Kalati F, Fashtami LA, Fateh M, Djavid GE. Treatment of oral lichen planus with photodynamic therapy mediated methylene blue: a case report. Med Oral, Patol Oral Cir Bucal. 2006; 11(2): E126-129.

[110] Kunte C, Erlenkeuser-Uebelhoer I, Michelsen S, Scheerer-Dhungel K, Plewig G. Treatment of therapy-resistant erosive oral lichen planus with extracorporeal photopheresis (ECP). J Dtsch Dermatol Ger 2005; 3(11): 889-94. 
[111] Guyot AD, Farhi D, Ingen-Housz-Oro S, et al. Treatment of refractory erosive oral lichen planus with extracorporeal photochemotherapy: 12 Cases. Br J Dermatol 2007; 156(3): 553-6.

[112] Marchesseau-Merlin AS, Perea R, Kanold J, Demeocq F, Souteyrand P, D'Incan M. Photopheresis: An alternative therapeutic approach in corticoresistant erosive oral lichen planus. Ann Dermatol Venereol 2008; 135(3): 209-12.

[113] Zhang J, Zhou G, Du GF, Xu XY, Zhou HM. Biologics, an alternative therapeutic approach for oral lichen planus. J Oral Pathol Med 2011; 40(7): 521-4.

[114] Xiong C, Li Q, Lin M, et al. The efficacy of topical intralesional BCG-PSN injection in the treatment of erosive oral lichen planus: A randomized controlled trial. J Oral Pathol Med 2009; 38(7): 551-
[115] Kalmar JR. Diagnosis and management of oral lichen planus. J Calif Dent Assoc 2007; 35(6): 405-11.

[116] Gandolfo S, Richiardi L, Carrozzo M, et al. Risk of oral squamous cell carcinoma in 402 patients with oral lichen planus: a follow-up study in an Italian population. Oral Oncol 2004; 40(1): 77-83.

[117] EAOM - Diagnostic and therapeutic protocol for the Management of Oral Lichen Planus in secondary care. Available from: http: //www.eaomedicine.com/webhtml/002/002/002.pdf.

[118] Cirillo N, Hassona Y, Pignatelli M, Gasparoto TH, Morgan DJ, Prime SS. Characterization of a novel oral glucocorticoid system and its possible role in disease. J Dent Res 2012; 91(1): 97-103.

[119] Cirillo N, Prime SS. Keratinocytes synthesize and activate cortisol J Cell Biochem 2011; 112(6): 1499-505 